\title{
Population fluctuations and regulation in great snipe: a time-series analysis
}

\author{
ANDREA KÖLZSCH*†, STEIN ARE SÆTHER \\ PEDER FISKEף, JACOB HÖGLUND† and JOHN ATLE KÅLÅS \\ *Department of Physics, AGNLD, University of Potsdam, Am Neuen Palais 10, 14469 Potsdam, Germany; \\ $\dagger$ Department of Ecology and Evolution, Population Biology and Conservation Biology, Uppsala University, \\ Norbyvägen 18D, 75236 Uppsala, Sweden; \$Netherlands Institute of Ecology (NIOO-KNAW), Centre for Terrestrial \\ Ecology, PO Box 40, 6666 ZG Heteren, the Netherlands; §Jönköping County Administrative Board, Hamngatan 4, \\ 55186 Jönköping, Sweden; $\mid$ Norwegian Institute for Nature Research, Tungasletta 2, 7004 Trondheim, Norway
}

\section{Summary}

1. During the last centuries, the breeding range of the great snipe Gallinago media has declined dramatically in the western part of its distribution. To examine present population dynamics in the Scandinavian mountains, we collected and analysed a 19-year time series of counts of great snipe males at leks in central Norway, 19872005.

2. The population showed large annual fluctuations in the number of males displaying at lek sites (range 45-90 males at the peak of the mating season), but no overall trend. 3. We detected presence of direct density-dependent mechanisms regulating this population. Inclusion of the density-dependent term in a Ricker-type model significantly improved the fit with observed data (evaluated with Parametric Bootstrap Likelihood Ratio tests and Akaike's Information Criterion for small sample size).

4. An analysis of (a number of a priori likely) environmental covariates suggests that the population dynamics were affected by conditions influencing reproduction and survival of offspring during the summer, but not by conditions influencing survival at the wintering grounds in Africa. This is in contrast to many altricial birds breeding in the northern hemisphere, and supports the idea that population dynamics of migratory nidifugous birds are more influenced by conditions during reproduction.

5. Inclusion of these external factors into our model improved the detectability of density dependence. This illustrates that allowing for external effects may increase statistical power of density dependence tests and thus be of particular importance in relatively short time series.

6. In our best model of the population dynamics, two likely density-independent offspring survival covariates explained $47 \cdot 3 \%$ of the variance in great snipe numbers (predation pressure estimated by willow grouse reproductive success and food availability estimated by the amount of precipitation in June), whereas density dependence explained 35.5\%. Demographic stochasticity and unidentified environmental stochasticity may account for the remaining $17 \cdot 2 \%$.

Key-words: density dependence, environmental covariates, Gallinago media, Parametric Bootstrap Likelihood Ratio test, population dynamics. 


\section{Introduction}

Many animal populations fluctuate around a stable size, seemingly regulated by some intrinsic or extrinsic mechanisms. Several factors have been suggested to regulate population density. They include internal factors such as demographic variability and social behaviour, and external factors such as weather, food supply, habitat quality and interactions with other species (Begon, Harper \& Townsend 1996; Newton 1998). Some of these factors may interact with each other or influence population growth variables in an enhanced, density-dependent way.

Density dependence is a widely discussed concept in population ecology (Pollard, Lakhani \& Rothery 1987; Murdoch 1994; Einum 2005) and can be defined as the 'actions of repressive environmental factors, collectively or singly, which intensify as the population density increases and relax as this density falls' (Huffaker \& Messenger 1964). Using the Ricker model (Ricker 1954) as a guideline, we are here concerned with negative, or regulatory, density dependence, where the annual growth rate $r_{t}$ is negatively related to the population size $N_{t}$. There is little doubt about the existence of such a regulatory process because many populations persist for long periods of time (Pollard et al. 1987). Much difficulty and controversy, however, prevails regarding how to quantify density dependence and how to statistically test for its presence (Wolda \& Dennis 1993; Fox \& Ridsdill-Smith 1995; Bjørnstad \& Grenfell 2001).

Recently, Lande et al. (2002) pointed out the importance of taking individual variability into account when studying population dynamics. To get a sufficient idea about demographic variability one needs several years of information on individual female survival, reproduction and dispersal. This kind of information may not always be available.

In this study we have worked with population counts of lekking great snipe Gallinago media (Latham, 1787). The spatial aggregation of great snipe males at lekking arenas provides ideal conditions for accurate estimation of male numbers. Females and nests on the other hand are much more difficult to collect information about.

As the data set that we used here is restricted to yearly male counts, we applied a simple time-series analysis approach to explore great snipe population fluctuations. The Parametric Bootstrap Likelihood Ratio (PBLR) test (Dennis \& Taper 1994) for density dependence provides a powerful and widely used, but controversial (Shenk, White \& Burnham 1998) method to develop a stochastic population model from simple census data. As this density dependence test uses clearly stated stochastic models it is possible to use an information theoretic approach of model selection. In addition to testing for density dependence on its own one can include external factors and test if they improve the fit of the model to population census data, and if their inclusion improves the detectability of density dependence (Rothery et al. 1997).
The aims of this study were to analyse the dynamics and possible mechanisms of regulation in a great snipe population. We checked for density dependence by using the Ricker population model, calculated AICcs (Akaike's Information Criterion for small sample size) and carried out several PBLR tests. To find the model that best explains the observed population fluctuations, we built and incorporated time series of some abiotic and biotic factors that we thought could influence great snipe numbers.

\section{Methods}

\section{GENERATING A POPULATION TIME SERIES}

We generated a population time series for the years 1987-2005 from field data for a great snipe population in a $6 \mathrm{~km}^{2}$ study area in Gåvålia, central Norway $\left(62^{\circ} 17^{\prime} \mathrm{N}, 9^{\circ} 36^{\prime} \mathrm{E}\right.$, see Løfaldli, Kålås \& Fiske 1992 and Fiske, Kålås \& Sæther 1994 for more details on the study area and general methods of the field work).

The time series represents numbers of territorial males in the study area that were generated from observational data from display arenas (leks). The study area supported four main lek arenas and male numbers on all leks were summed to give a population size estimate for the whole study area. In some years smaller and more irregular leks turned up within the study area, resulting in data from a total number of 12 leks included in this study. Field work and data collection were supervised by one of us (JAK) during the whole study period, thus keeping variation of data quality and census error at a minimum. Direct counts of males (> 90\% marked with unique colour ring codes) that displayed at leks in the peak mating period (27 May-5 June) were available for most of the leks in the actual study area (82 of the 100 included lek-years).

For time-series construction we used only highquality counts (where observers spent several hours in hides at a lek and were sure that the count was accurate to \pm 1 individual that night). If several such counts were available from one lek during the actual time period the average number was used. In some years during 198796 there were active leks with no high-quality counts during 27 May-5 June ( $n=17$ lek years). For 15 of these we used counts from just before or after this period. One of the main lekking arenas was not observed in 1987 and 1988. For these 2 years we generated an estimate of the number of territorial males for this lek by multiplying proportional changes in numbers on the other leks with counts in the considered lek from later years. From 1997 onwards there were no birds present at one of the main previous leks during the period 1987-96 (ØS). In 1998 we found a new lek (SAL) only c. $700 \mathrm{~m}$ from the lek that terminated in 1997. This lek has later been used annually, while no lekking birds have been observed at the ØS lek. We find it reasonable to assume that the SAL lek replaced the ØS lek in 1997. Therefore, we estimated the number of 
territorial males for SAL for 1997 by multiplying proportional changes in numbers on the other leks with counts on SAL from 1998 and with counts on ØS from 1996, and taking the mean value of these two estimates.

Even if there is some movement between leks (Sæther, S.A. and Kålås, J.A., unpublished data), the number of territorial males at each lek is usually very stable during the period 27 May-5 June and makes this period appropriate for counting males. This is illustrated by counts from two of the included leks, $\mathrm{AH}$ and $\mathrm{HN}$, where we have many high-quality counts during this 10-day period for most of the years (Fig. 2b,c). We tested the stability assumption by comparing withinand between-year variation for those two leks.

\section{POPULATION STABILITY}

In the following we assume that the series of territorial males in our population of great snipe is representative for the general population fluctuations in our study area.

For a population to be considered stable there should be no trend in the time series and population size deviations should be bounded. With a linear regression of $N_{t}$ on time we checked if the time series of territorial males showed indications of a trend. Because population fluctuations that show no trend are limited if their variance is small and yearly population growth rates $r_{t}=\ln \left(N_{t+1} / N_{t}\right)$ are negatively autocorrelated (Royama 1992), we calculated autocorrelation coefficients of several lags for $r_{t}$.

\section{DENSITY DEPENDENCE}

To examine if population growth was density-dependent, we tested if the magnitude of intrinsic population growth was directly (or in a delayed manner) dependent on population size. This assumes that population growth was not constant, but limited by resource availability or other factors.

Our model investigations of population densities are based on the PBLR test of Dennis \& Taper (1994) that tests for direct density dependence. This test uses a stochastic form of the Ricker model to describe population fluctuations

$N_{t+1}=N_{t} \exp \left(a+b N_{t}+\sigma Z_{t}\right)$

eqn 1

Thus, $r_{t}=a+b N_{t}+\sigma Z_{t}$. The parameters $a, b$ and $\sigma$ are constants and $Z_{\mathrm{t}}$ is assumed to be independent, and drawn from a standard normal distribution. Density dependence is confirmed if the test indicates the incorporation of $b<0$. We present PBLR test results, the likelihood ratio and its $P$-value from bootstrap samples to indicate significance and strength of possible direct density dependence in the observed series. We also compared AICc values (Sugiura 1978) calculated for the density-independent and the density-dependent model. The AIC adjusted for small sample size has a bias correction term to correct for effects of small sample size such as our data of 19 yearly counts. To quantify the quality of the model fit we calculated the coefficient of determination $R^{2}$ that provides the proportion of variation in the data that can be attributed to the explaining variables in a model. Here, a proportion of $R^{2}\left(N_{t}\right)=b^{2} \operatorname{Var}\left(N_{t}\right) / \operatorname{Var}\left(r_{t}\right)$ of variation in the population growth rates can be attributed to density dependence. We present plots of the regression model for parameter estimations and some simulated trajectories of the performances of the density-independent $(b=0)$ and density-dependent $(b<0)$ model to give visual help in the interpretation of test results.

We examined the order of population density dependence patterns, using modified Partial Rate Correlation Functions (PRCF) that have been developed to explore biological systems dynamics (Berryman \& Turchin 2001). Considering the model structure of the Ricker model (relating $r_{t}$ to $N_{t}$ ), which is used throughout this study, we calculated PRCFs as the partial correlation coefficients between $r_{t}$ and $N_{t}$. Because of the relative shortness of our time series, the modified PRCFs were only calculated up to lag 5 for each series. Indications of delayed density dependence were in addition tested with AICcs and a PBLR test for the modified model

$N_{t+1}=N_{t} \exp \left(a+b N_{t-i}+\sigma Z_{t}\right)$

eqn 2

\section{GENERATING TIME SERIES OF EXTERNAL FACTORS}

Populations can be regulated by different mechanisms. Environmental factors and resource availability, social behaviour, species interactions and demographic differences may cause or alter density-dependent fluctuation patterns (Begon et al. 1996; Lande, Engen \& Sæther 2003; Turchin 2003). For the studied great snipe population we have no relevant female fecundity information available, but yearly climate conditions and an index of biological interactions (predation) of great snipe could be incorporated into our model. To minimize problems of multiple testing we selected a priori a small number of potentially relevant external factors, presented below.

\section{Breeding season food availability}

To account for food availability in the breeding area, we generated two time series of precipitation measures. Great snipe mainly feed on earthworms during the breeding season (Løfaldli et al. 1992). More rain is assumed to improve feeding conditions for great snipe, because earthworms are in the top ground layer when it is wet and moist ground is easier to penetrate in order to capture them. Daily precipitation amounts from a weather station in Fokstua $\left(62^{\circ} 07^{\prime} \mathrm{N}, 9^{\circ} 17^{\prime} \mathrm{E}\right)$, c. $20 \mathrm{~km}$ south-west of the study area, were provided by Norway's Meteorological Institute (www.met.no). We calculated two precipitation time series by summing 
daily amounts of rain; first for the period when great snipe are incubating (5-24 June, 'incubation rain') and then for the period when hatchlings are growing and highly vulnerable (21 June-20 July, 'growth rain').

\section{Breeding season phenology}

Yearly recorded dates of when the surface of Lake Gåvålivatn in the study area was $>90 \%$ free of ice were used to create a time series of phenology ('ice index'). For 1987 such a date was not available. Therefore, we estimated it from a regression with Fokstua snow data (Linear regression: $F_{1,12}=24 \cdot 82, P=0 \cdot 0002, R^{2}=0 \cdot 623$ ).

\section{Breeding season North Atlantic Oscillation (NAO)}

The NAO index has been used in several population studies to account for general climatic conditions in Europe. From monthly NAO indices that were obtained from the Climatic Research Unit of the University of East Anglia in Norwich, UK (Jones, Jonsson \& Wheeler 1997; www.cru.ac.uk/ftpdata/nao.dat) we calculated a mean NAO index for May-August, the time that great snipe breed in Norway ('NAO').

\section{Breeding season predation}

We acquired a time series of willow grouse Lagopus lagopus (Linnaeus, 1758) reproduction ('grouse reproduction') in Einunndalen, situated c. 20-30 km east of Gåvålia. These reproduction estimates (number of chicks per two adults) were based on the age composition of birds shot by local hunters (aged based on pigmentation of collected wing feathers; see Bergerud, Peters \& McGrath 1963). Willow grouse and great snipe have common predators of their offspring. We therefore assumed that low grouse reproduction indicates high predation pressure on great snipe, particularly on eggs and offspring. A positive relationship between $r_{t}$ and grouse reproduction could alternatively be due to the use of the same habitat and the two species being affected by similar climatic and biological limitations. However, because grouse chicks feed on insects, whose abundance would be affected by weather conditions in the opposite rather than parallel direction to earthworms, this would lead to a negative relationship between $r_{t}$ and grouse reproduction.

\section{Wintering food availability}

Food availability in their wintering habitats in Africa may also influence great snipe survival. We calculated such time series from global precipitation data provided by the Global Precipitation Climatology Centre (GPCC) of Germany's National Meteorological Service 'Deutscher Wetterdienst' (Rudolf et al. 2003; http:// gpcc.dwd.de). Monthly precipitation measures were available for 1986-2004 based on quality controlled data from a large number of weather stations. The data

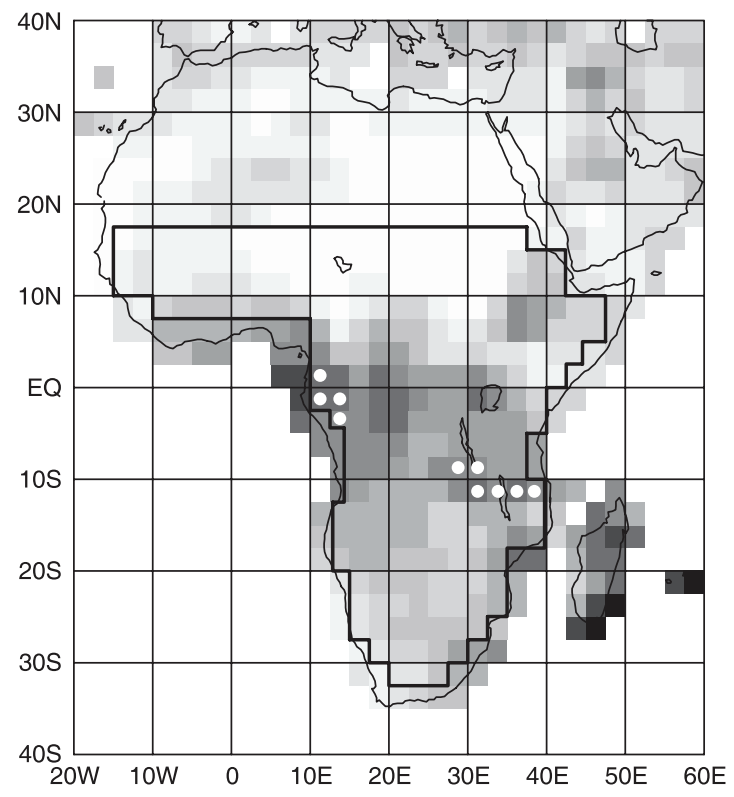

Fig. 1. The bold line indicates the selected area of African precipitation grids used in this study. The small white dots indicate the selected maximum precipitation grids for March, used to construct the African precipitation time series for this month. As an example, the different shading represents precipitation amounts in each 2.5 $5^{\circ}$ grid for March 2005.

are grid-related and we used grids with a resolution of $2 \cdot 5^{\circ}$ latitude/longitude. From the large amount of grid data we selected the grids that were relevant as great snipe wintering areas, i.e. all grids south of the Sahara that were $>90 \%$ situated on land $(n=245$, Fig. 1$)$. For 1986-2004, medians of monthly precipitation were calculated for each of these grids. We summed the precipitation amounts of the 10 grids of maximal median rain: (1) in September and October ('arain Sep/Oct') to represent ground wetness for the time just after great snipe have arrived to their first wintering areas; (2) in December and January ('arain Dec/Jan') as the mid winter maximum precipitation; and (3) in March and April ('arain Mar/Apr') to represent food availability to great snipe just before they migrate back to their breeding grounds. Using the selected grids as preliminary areas of great snipe presence, we accounted for the suggestion that great snipe follow the progression of the African wet season during the winter (Massoli-Novelli 1988) and may be found in areas of maximal precipitation.

\section{INFLUENCES OF EXTERNAL FACTORS ON POPULATION GROWTH}

First, we conducted a preliminary analysis of relations between great snipe population fluctuations and the proposed external factors using simple correlations. In correspondence to the model, we looked at correlations between population growth rates (rather than population size estimates) and external factors. This is reasonable, because environmental factors often influence rates of population change directly. 
Standard correlation tests assume that population time series are stationary and that external factors are not autocorrelated. Assuming population stability and considering the nature of most of the used external factors (local weather conditions should be independent between the years), these assumptions seem reasonable. To test this independence assumption we checked for autocorrelations in the external factor time series. For all time series of external factors cross-correlations with $r_{t}$ of several lags were also calculated.

Correlation test results can be affected by correlations between explanatory variables. Therefore, we calculated correlations between all pairs of our external factors. The strength of correlations between population growth rates and external factors may also depend on the structure of any existing density dependence in the population fluctuations (Royama 1992). We thus continued with our primary approach of directly modelling population dynamics where density dependence can be taken into account, and included external factors into model (eqn 1).

We modified our density dependence model to test for the influence of external factors on population dynamics. Each covariate $\left(W_{i, t}\right)$ was directly incorporated into the model, one at a time

$N_{t+1}=N_{t} \exp \left(a+b N_{t}+c_{i} W_{i, t}+\sigma Z_{t}\right) ; i=1,2, \ldots, 8$ eqn 3

So, we explicitly attributed a part of the environmental stochasticity that is represented by $\sigma Z_{t}$ to an external factor. To select among models we calculated AICc values and PBLR $P$-values of likelihood ratios for each external factor and decided which factors significantly improved the model fit.

FINAL MODELS OF GREAT SNIPE POPULATION FLUCTUATIONS

To decide on an appropriate final model for the great snipe time series, several possible models were constructed, their AICcs calculated and PBLR tests conducted. Using the results of our previous model analyses, we examined if certain combinations of external variables improved model performance. In each step we only kept additional variables in the model if AICcs and PBLR test results showed a significant improvement of the model's performance. We present the structure, parameter estimates, goodness of fit and some simulated trajectories of the final model to visualize its performance. Each trajectory represents a model realization of different stochastic processes $Z_{t}$.

\section{DENSITY DEPENDENCE ALLOWING FOR EXTERNAL EFFECTS}

Using the final model with its external factors we could again test for density dependence in our population of great snipe following the ideas of Rothery et al. (1997), who suggested that including exogenous factors may increase the power to detect density dependence. In line with our previous analyses AICc and PBLR test statistics were calculated for the final model including and excluding the density dependence term $b N_{t}$.

\section{MEASUREMENT ERROR AND MODEL EVALUATION}

Measurement error may cause spurious detection of density dependence in ecological time series (Shenk et al. 1998; Freckleton et al.2006). One indicator of the magnitude of measurement error in fitted data is the autocorrelation in the model residuals (Dennis et al. 2006). Therefore, we examined if the residuals of our first density dependence model (eqn 1) and the final model (eqn 3) show autocorrelation. In addition, we run a simulation suggested by Freckleton et al. (2006) to quantify the effects of possible census error on our results. First, we fitted a density-independent model (eqn $3, b=0$ ) to our data obtaining a strictly densityindependent time series. Our lek counts were accurate to \pm 1 individual. Thus, with four main leks in most years, possible measurement error was restricted to the interval $[-4,4]$ for each year. We simulated 1000 errorprone data series by adding random, possible census errors to the density-independent time series. PBLR and AICc values were calculated for each, and we examined if possible measurement errors were sufficient to generate the levels of density dependence observed in our data.

The Ricker model assumes that there are linear associations between population growth $r_{t}$ and: (1) population size $N_{i}$; (2) each external factor; and (3) stochasticity. This form of relationship may not apply, and explanatory factors could interact with each other as well. As a first-order approximation, however, linear equations are useful to describe population dynamics even of nonlinear behaviour.

For model evaluation and to account for the appropriateness of the general model assumptions of $Z_{t}$ being independent and standard normally distributed, we checked if model residuals are autocorrelated and follow a normal distribution. We also examined if squared residuals from the data depend on the data, i.e. the population size, thus verifying if the variance of $Z_{t}$ is constant.

All analyses were conducted using R (Ihaka \& Gentleman 1996).

\section{Results}

GREAT SNIPE POPULATION TIME SERIES AND STABILITY

The generated population time series (Fig. 2a) is characterised by the number, direction and strength of its fluctuations. $N_{t}$ has a mean of $65 \cdot 3$ males with a standard deviation of $10 \cdot 4$, range between $44 \cdot 9$ and 

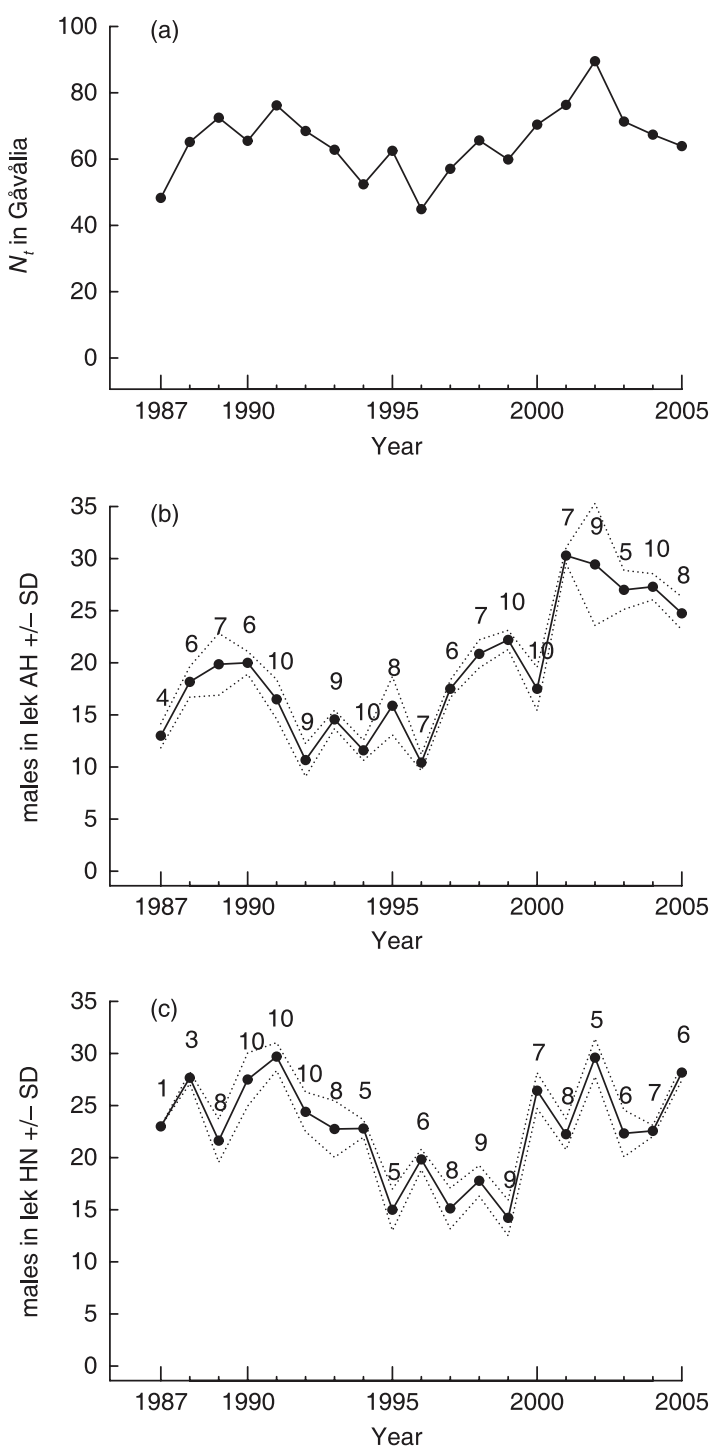

Fig. 2. Obtained time series of territorial great snipe males in the whole study area (a) and the two main leks AH (b) and HN (c). The dotted lines in (b) and (c) give $N_{t} \pm \mathrm{SD}$ of the lek counts, thus accounting for the variability of male numbers in the main mating period due to movement between leks.

$89 \cdot 5$ males, and a coefficient of variation of $0 \cdot 159$. This range is relatively wide, the maximum number being twice the minimum. The annual population growth rate was close to zero with a mean of 0.0156 and a range from -0.33 to $0 \cdot 30$. The number of years of population increase ( $n=9$ years) was the same as the number of years of population decrease, and mean population sizes before an increase $(\bar{N}=60 \cdot 0)$ or decrease $(\bar{N}=$ $70 \cdot 7)$, differed. These characteristics indicate stability and regulation. The numbers of years of population increase and decrease are balanced and there are no runs of more than 3 years of successive increase or decrease (Fig. 2a). Years of especially high population density seem to be followed by strong decreases in individual numbers. A linear regression of $N_{t}$ on time does not suggest a trend in the population time series $\left(F_{1,17}=1 \cdot 674, P=0 \cdot 21, R^{2}=0 \cdot 090\right)$.
The within-year coefficient of variation for the main mating period (27 May-5 June) varied for lek HN between 0.021 and 0.133 (17 years with more than 4 days of counts during this period), and for lek $\mathrm{AH}$ between $0 \cdot 025$ and $0 \cdot 149$ (all 19 years with more than 4 days of counts) with one outlier of $0 \cdot 199$ in 2002. A Kruskal-Wallis test confirms that within-year variation was less than between-year variation $\left(\chi_{18}^{2}=136 \cdot 33\right.$, $P<0.001$, and $\chi_{18}^{2}=115.42, P<0.001$ for $\mathrm{AH}$ and $\mathrm{HN}$, respectively). The within-year standard deviations (Fig. 2b,c) emphasize this finding. Thus, in the chosen time period, male numbers are relatively stable and our data are appropriate for the described analysis.

Autocorrelation coefficients $r_{i}$ for lags $i=1,2, \ldots, 5$ of yearly $r_{t}$ values for our time series of territorial males are $r_{1}=-0.275, r_{2}=0.089, r_{3}=0.032, r_{4}=-0.054$ and $r_{5}=-0 \cdot 250$. None of these sample autocorrelation coefficients of $r_{t}$ were significant, indicating that population fluctuations are large and population size not very strongly regulated. However, direct density dependence and population stability are supported by a negative first order autocorrelation coefficient $r_{1}$ of $r_{t}$, as well as by $\bar{r}_{t} \approx 0$.

\section{DENSITY DEPENDENCE}

The AICc for the density-dependent model (-12.68) was distinctly lower than for the density-independent model (-7.79), supporting the presence of density dependence in our time series. PBLR test results for density dependence were not quite significant $(P=$ $0 \cdot 055$, see Table $2 \mathrm{a}$ ). However, power is limited as the time series is quite short. Density dependence alone explains $R^{2}\left(N_{t}\right)=33.4 \%$ of the variation in population growth. The maximum likelihood estimate $\hat{b}=-0 \cdot 010$ in model (eqn 1) is negative, which indicates regulatory density dependence. Because of the relative shortness of our population time series and as most biological mechanisms that we considered would work with lag 1 on population size or directly on population growth, we interpret these results as indications of density dependence, even if perhaps weak. The visualizations of density dependence testing in Fig. 3 support this conclusion. The pattern of successive yearly relations between $r_{t}$ and $N_{t}$ in Fig. 3a reveals a cyclicity of population sizes and the density-dependent model (Fig. 3c) gives better, less widely fluctuating, fits of model trajectories to the observed time series than the null model in Fig. 3b.

Checking for the order of any density dependence (direct density dependence: order $=1$; lagged density dependence: order $>1$ ) in the studied great snipe population revealed a significant negative partial rate correlation coefficient of order $1(\mathrm{PRCF}[1]=-0 \cdot 593$, $P=0.009)$. This is in line with AICc model selection and PBLR test results. In addition there was an indication of a negative lag 5 partial rate correlation coefficient $(\mathrm{PRCF}[5]=-0 \cdot 545, P=0 \cdot 098)$, but neither AICc $=$ -2.99 (compared with AICc $=-7.79$ for the density- 

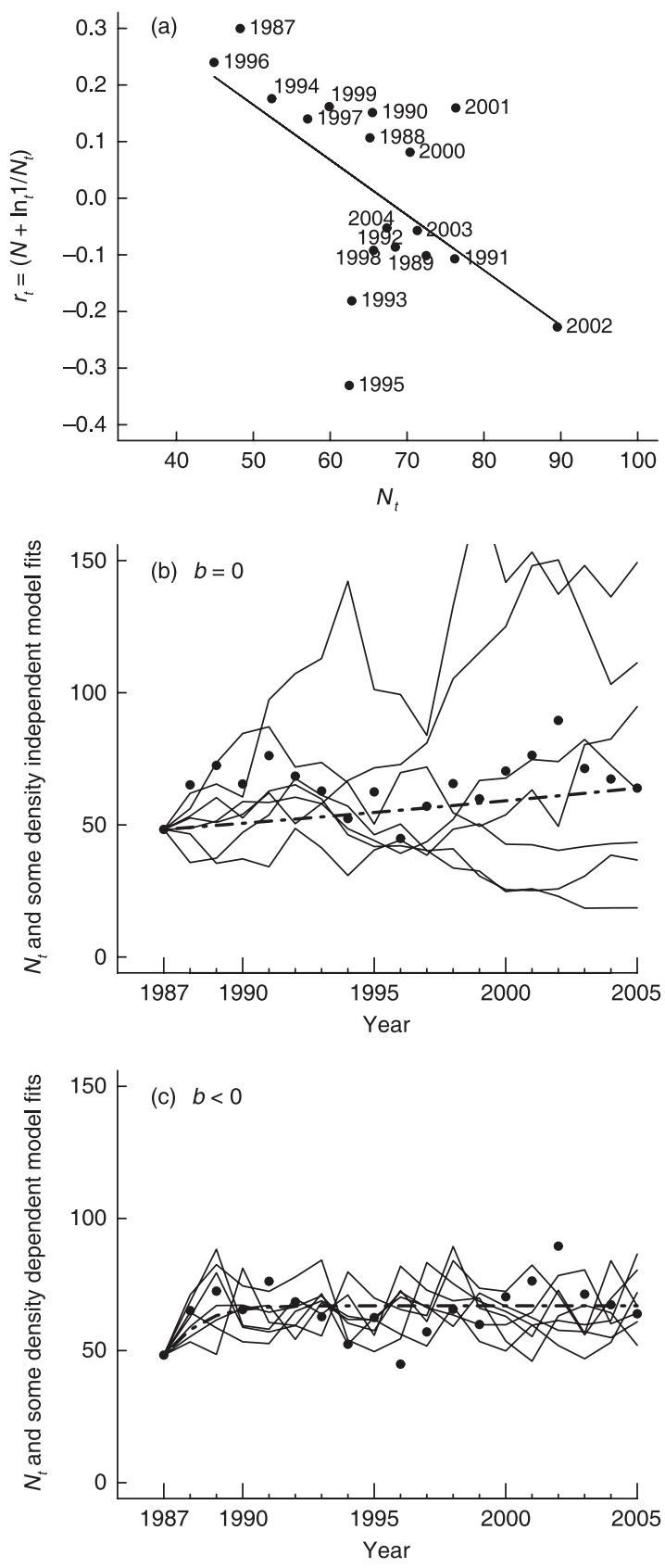

Fig. 3. Parametric Bootstrap Likelihood Ratio test results for density dependence of territorial male great snipe in Gåvålia. (a) shows the regression of the growth rate $r_{t}$ and the population size estimate $N_{t}$, from which parameters for the model were estimated. In (b) some model trajectories of the densityindependent null model and their fit on the data are presented. Model trajectories for the density-dependent model are shown in (c). The black dots represent yearly data points and the dashed, bold lines indicate each model's deterministic fit with $\sigma=0$.

independent model) nor a PBLR test $(\mathrm{LR}=0.596$, $P=0 \cdot 392)$ support the suggestion of such lagged density dependence.

\section{EXTERNAL FACTORS}

None of the external factors time series showed signs of autocorrelation. Two cross-correlation coefficients between $r_{t}$ and external factors (see coefficients for lag 0 and lag +1 in Table 1 ) were significant. There was a positive correlation between $r_{t}$ and growth rain (lag 0 , $r=0.726$ ), which suggests that food availability during the offspring growth period affects great snipe numbers in the following year. Furthermore, there was a negative lag +1 cross-correlation coefficient between $r_{t}$ and grouse reproduction of $r_{+1}=-0 \cdot 612$, supporting the idea that predation (presumably on offspring) affects great snipe population growth.

Two correlations between the external factors time series were significant: $r=-0.585$ between the ice index and arain December/January $(P=0.011)$ and $r=0.522$ between growth rain and arain March/April $(P=$ $0 \cdot 026$ ). As both pairs of climate variables are spatially separated we assume that these correlations do not confound our analysis.

Results of various PBLR tests and AICc values for model improvement with each external variable show that all significant relations of $r_{t}$ with a covariate are positive $\left(c_{i}>0\right.$ for $i=2,5$; Table 2$)$. Population growth rates of great snipe seem to be related to food availability in the growth period of the offspring ( $i=2$, growth rain) and predation during the breeding season $(i=5$, grouse reproduction). Thus, conditions during reproduction seem to be more important than variation in wintering conditions for great snipe population dynamics.

\section{FINAL MODELS OF GREAT SNIPE POPULATION FLUCTUATIONS}

Our best model to explain fluctuations in the time series of territorial great snipe males is of the form

$N_{t+1}=N_{t} \exp \left(a+b N_{t}+c_{2} W_{2, t}+c_{5} W_{5, t}+\sigma Z_{t}\right)$

eqn 4

where $W_{2, t}$ represents growth rain and $W_{5, t}$ grouse reproduction. The maximum likelihood estimates for the parameters are $\hat{a}=-0 \cdot 020(\mathrm{SE}=0 \cdot 145), \hat{b}=-0.009$ $(\mathrm{SE}=0.002), \hat{c}_{2}=0.005(\mathrm{SE}=0.001), \hat{c}_{5}=0.103(\mathrm{SE}=$ $0 \cdot 029$ ) and $\hat{\sigma}=0.073$ (for AICcs and PBLR test results see Table 2c). Of the overall model variance in $r_{t}$, density dependence explains $R^{2}\left(N_{t}\right)=35 \cdot 5 \%$, precipitation between 21 June and 20 July $R^{2}\left(\mathrm{~W}_{2}\right)=33 \cdot 1 \%$ and predation, indexed by grouse reproduction, $R^{2}\left(\mathrm{~W}_{5}\right)=$ $14 \cdot 2 \%$. Thus, the deterministic part of the model explains $82.8 \%$ of the population growth variation. Model trajectories of this final model revealed a good fit with the observational data (see Fig. 4). The remaining $17 \cdot 2 \%$ of the variation in $r_{\mathrm{t}}$ may be accounted for by demographic stochasticity and some remaining, unknown environmental stochasticity.

\section{DENSITY DEPENDENCE ALLOWING FOR EXTERNAL EFFECTS}

Results of the additional density dependence test allowing for the selected environmental effects (see 
Table 1. Cross-correlation coefficients of external factors on the annual population growth rate $r_{t}(\operatorname{lag} 0)$ and $r_{t+1}(\operatorname{lag} 1)$ of great snipe. Because 'arain September/October' values were not normally distributed, the Spearman correlation coefficient is reported for this variable instead of the Pearson correlation coefficient

\begin{tabular}{|c|c|c|}
\hline External factor $\left(W_{i t}\right)$ & Correlation to $r_{t}$ & Correlation to $r_{t+1}$ \\
\hline Incubation rain & $0 \cdot 33(P=0 \cdot 17)$ & $0.35(P=0 \cdot 17)$ \\
\hline Growth rain & $0 \cdot 73(P=0 \cdot 001)$ & $-0 \cdot 13(P=0 \cdot 63)$ \\
\hline Ice index & $0 \cdot 14(P=0 \cdot 57)$ & $-0.07(P=0.78)$ \\
\hline NAO & $-0.03(P=0.90)$ & $-0 \cdot 37(P=0 \cdot 15)$ \\
\hline Grouse reproduction & $0 \cdot 35(P=0 \cdot 15)$ & $-0 \cdot 61(P=0 \cdot 009)$ \\
\hline Arain September/October & $-0.02(P=0.94)$ & $0 \cdot 11(P=0 \cdot 69)$ \\
\hline Arain December/January & $0.08(P=0.77)$ & $-0 \cdot 17(P=0 \cdot 51)$ \\
\hline Arain March/April & $0 \cdot 36(P=0 \cdot 14)$ & $-0 \cdot 22(P=0 \cdot 39)$ \\
\hline
\end{tabular}

Incubation rain $=$ the amount of precipitation in the study area during the incubation period; growth rain = precipitation during the chick growth period; ice index $=$ phenology; $\mathrm{NAO}=$ the North Atlantic Oscillation index; grouse reproduction $=$ the proportion of juvenile willow grouse; arain $=$ the precipitation in the African wintering areas in the specified months.

Table 2. Comparison of the proposed models of great snipe population dynamics, using AICc and PBLR test results

\begin{tabular}{|c|c|c|c|c|c|}
\hline & & PBLR & st results & & \\
\hline & Model $\left(\mathrm{H}_{1}\right)$ & $\mathrm{AICc}$ & $\mathrm{H}_{0}$ & LR & $P$ \\
\hline (a) & DID & $-7 \cdot 79$ & & & \\
\hline & DD & $-12 \cdot 68$ & DID & $0 \cdot 02$ & $0 \cdot 055$ \\
\hline (b) & $\mathrm{DD}+\mathrm{W}_{1}$ & $-10 \cdot 91$ & DD & $0 \cdot 45$ & $0 \cdot 26$ \\
\hline & $\mathrm{DD}+\mathrm{W}_{2}$ & $-25 \cdot 26$ & DD & $\mathbf{0} \cdot 00$ & $<0.001$ \\
\hline & $\mathrm{DD}+\mathrm{W}_{3}$ & $-9 \cdot 83$ & DD & $0 \cdot 77$ & 0.53 \\
\hline & $\mathrm{DD}+\mathrm{W}_{4}$ & $-9 \cdot 97$ & DD & $0 \cdot 72$ & $0 \cdot 48$ \\
\hline & $\mathrm{DD}+\mathrm{W}_{5}$ & $-16 \cdot 68$ & DD & $0 \cdot 03$ & $0 \cdot 015$ \\
\hline & $\mathrm{DD}+\mathrm{W}_{6}$ & $-9 \cdot 45$ & DD & $0 \cdot 94$ & $0 \cdot 72$ \\
\hline & $\mathrm{DD}+\mathrm{W}_{7}$ & $-12 \cdot 15$ & DD & $0 \cdot 24$ & $0 \cdot 15$ \\
\hline & $\mathrm{DD}+\mathrm{W}_{8}$ & $-12 \cdot 47$ & DD & $0 \cdot 21$ & $0 \cdot 11$ \\
\hline (c) & $\mathrm{DD}+\mathrm{W}_{2}+\mathrm{W}_{5}$ & $-32 \cdot 79$ & $\mathrm{DD}+\mathrm{W}_{2}$ & $\mathbf{0} \cdot \mathbf{0 0}$ & $<0.001$ \\
\hline (d) & $\mathrm{W}_{2}+\mathrm{W}_{5}$ & $-17 \cdot 49$ & & & \\
\hline & $\mathrm{DD}+\mathrm{W}_{2}+\mathrm{W}_{5}$ & $-32 \cdot 79$ & $\mathrm{~W}_{2}+\mathrm{W}_{5}$ & $0 \cdot 00$ & $<0.001$ \\
\hline
\end{tabular}

AICc is the Akaike's Information Criterion corrected for small sample size, PBLR test is the Parametric Bootstrap Likelihood Ratio test. Model abbreviations are: DID, densityindependent model; DD, density-dependent term included; $\mathrm{W}_{1}$, precipitation amount during the incubation period; $\mathrm{W}_{2}$, precipitation amount during the chick growth period; $\mathrm{W}_{3}$, index of phenology by ice cover duration; $\mathrm{W}_{4}$, mean breeding period $\mathrm{NAO} ; \mathrm{W}_{5}$, reproduction of willow grouse as index of predation; $\mathrm{W}_{6}$, precipitation in African wintering areas during September/October; $\mathrm{W}_{7}$, precipitation in African wintering areas during December/January; $\mathrm{W}_{8}$, precipitation in African wintering areas during March/April. AICc-values correspond to the stated $\mathrm{H}_{1}$-Model, and sample likelihood ratios $\left(\mathrm{LR}=\mathrm{L}\left(\mathrm{H}_{0}\right) / \mathrm{L}\left(\mathrm{H}_{1}\right)\right)$ and $P$-values are presented together with the respective null model $\left(\mathrm{H}_{0}\right)$ that each model was nested within. Important results are highlighted in bold.

Table $2 \mathrm{~d}$ ) are very clear. When including growth rain and grouse reproduction, density dependence emerges as an important component of the model and thus of population dynamics of the studied great snipe population.

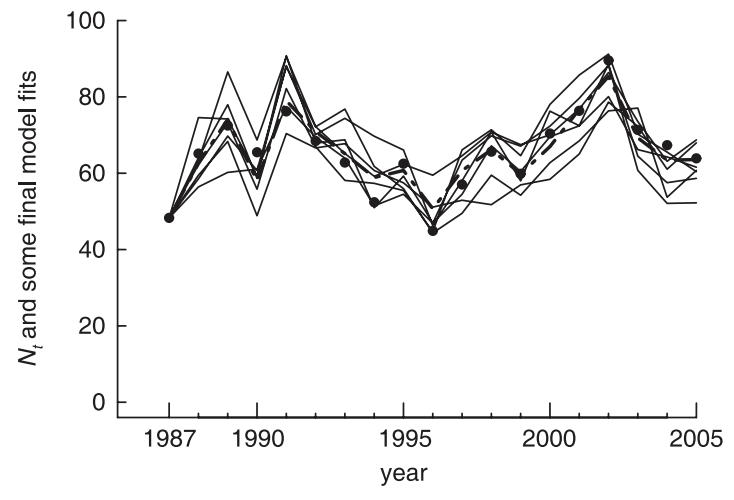

Fig. 4. Some trajectories of the final model fit on the data (black dots). Each realization uses a different stochastic process $Z_{t} \sim N(0,1)$, thus the whole picture shows some of the range of the model fit. The dashed, bold lines indicate the final model's deterministic fit with $\sigma=0$.

\section{MEASUREMENT ERROR AND MODEL EVALUATION}

Residuals of the simple density dependence model (eqn 1) did not show significant autocorrelation. Thus, measurement error seems to be small (Dennis et al. 2006). The final model allowing for density dependence and weather effects, however, reveals autocorrelation in its residuals (e.g. $r_{1}=-0 \cdot 461$ ). This indicates that mechanisms not accounted for by us (e.g. demographic stochasticity) may be at work in the population, controlling residual dynamics. Therefore, autocorrelation of the residuals may not be a reliable indicator for measurement error in this study. Our sensitivity analysis using simulated census errors suggested that our levels of measurement error cannot account for the density dependence. Only $2 \cdot 3 \%$ of the 1000 simulated errorprone density-independent time series showed PBLR $P$-values smaller than $0 \cdot 05$. Similarly, of the simulated AICc values only $2 \%$ showed indications of spurious density dependence (AICc(DD) < AICc(DID)).

The residuals of the final model did not significantly deviate from a normal distribution (Shapiro-Wilk tests: $W=0.974, P=0.87)$, but they seem to be autocorrelated (see above). Squared residuals did not appear to depend on numbers of observed males (linear regression: $F_{1,16}=0.008, P=0.93$ ), thus it is likely that $\operatorname{Var}\left(Z_{t}\right)$ is constant.

\section{Discussion}

The population of great snipe in Gåvålia shows characteristics of stability and several populationlimiting factors that potentially are density-dependent may be at work in the studied population.

Great snipe are habitat and food specialists (Løfaldli et al. 1992; Kålås, Fiske \& Höglund 1997) and therefore food resources may be limited on the breeding grounds. However, it is difficult to know what the exact mechanisms of food limitation are. Although food availability 
in the offspring growth period is likely to predominately affect the survival of offspring, it could also influence the survival of adults, because the adult males have to replenish their fat resources for migration after a period of highly energy demanding display activity (Höglund, Kålås \& Fiske 1992). Because of its strong direct correlation with $r_{t}$, food availability in the offspring growth period seems to influence survival in a densityindependent way. This could be because the principal food source of great snipe, earthworms (Løfaldli et al. 1992) move downwards in the ground when it gets dry and may become inaccessible for great snipe. This would act homogeneously throughout a large area, when there is not much rain, and thus independent of population density. If such poor environmental conditions occur in several consecutive years, the observed large fluctuations of population size between years indicate that the population could reach critically low numbers.

Predation on offspring of different prey species is an important process in the rather simple food web of Scandinavian alpine ecosystems (Wiklund, Kjellén \& Isakson 1998). A positive relationship between $r_{t}$ and grouse reproduction in the Ricker model and a negative correlation between grouse reproduction and $r_{t+1}$ suggest that predation on offspring is important in our studied great snipe population. Because grouse are present in the habitat year round and provide high biomass prey for top predators, their numbers may influence predator population dynamics. High grouse numbers may lead to population increase in the predator species, and a high predator density in the year following a grouse population peak may lead to high predation pressure on great snipe and thus decreased population growth in such years.

One or several density-dependent mechanisms appear to be important in regulating the studied great snipe population. In particular, the existence of direct density dependence (especially when allowing for external effects) seems to be confirmed. Possible mechanisms directly affecting population growth parameters are density-dependent reproduction, predation on breeding birds, eggs and hatchlings, interference competition, competition for food and climate conditions that influence survival. However, one has to keep in mind that this analysis is based on a time series of $n=19$. Thus, only the simplest mechanisms controlling population dynamics can be detected.

Our results suggest that great snipe population dynamics are more affected by environmental conditions on the breeding grounds (the 'tap' hypothesis, Sæther, Sutherland \& Engen 2004) than by conditions during the winter in Africa (the 'tub' hypothesis, Lack 1954). This is in contrast to many migratory birds breeding in the Northern temperate zone that behave according to the 'tub-hypothesis', but supports the suggestion that nidifugous birds, like the great snipe, are more susceptible to conditions during the breeding season than are altricial birds (Sæther et al. 2004).
However, we do not know where in Africa great snipe breeding in Norway stay during the winter. We have assumed here that great snipe reside in maximum precipitation areas (Massoli-Novelli 1988), and obviously a more detailed knowledge about where the birds spend the winter would give better and more reliable results. In particular, information about where great snipe stay just before their migration back to the breeding grounds would be useful, because feeding conditions at this time should impact the energy reserves needed for successful migration.

Population dynamics patterns, especially of longlived species, are often influenced by life history and demographic stochasticity in the population (Lande et al. 2002). Our model accounts for environmental stochasticity. To include demographic stochasticity into a model, information about the reproduction of females is needed. As this is not available for the studied great snipe population, we can only speculate that some of the variability that is not explained by density dependence and external factors is due to variation in life-history parameters. However, it may be appropriate to ignore any explicit measures of demography because great snipe have a relatively simple life history (reproduction starts when 1 year old, and the clutch size is fixed at four eggs).

Tests of density dependence, and especially their applicability to ecological population time series in the presence of measurement error, have recently been debated (Shenk et al. 1998; Freckleton et al. 2006). Shenk et al. (1998) concluded that measurement error inflates type I error rates for density dependence tests, including the PBLR test, thus invalidating this method to correctly detect density dependence in the presence of census error. Only if sampling error is proportional to $N_{\mathrm{t}}$ and variation small, would the claimed robustness of this test (Dennis \& Taper 1994) hold. Even if this is not strictly fulfilled in our case, we still consider the PBLR test the most appropriate for our simple census data. This is because it clearly states the models it works with, it does not require additional information of life history or state-space, and one can easily allow for environmental effects (thus increasing power; Rothery et al. 1997). Census error was estimated during the field work and by conducting a final sensitivity analysis we found that a far higher level of measurement error than estimated would be needed to discount our results of density dependence testing as spurious. Therefore, we claim that our main conclusions hold, even in the presence of small census errors.

Debate prevails about the general usefulness of timeseries analyses in population dynamics and the distinction of density-dependent and density-independent mechanisms in population dynamics analyses (Wolda \& Dennis 1993; Leirs et al. 1997; Turchin 1999). In our model, density-dependent as well as density-independent mechanisms were accounted for and both seem to be important in this great snipe population. By using a simple approach of linear modelling of a population 
time series, we were able to explain a large amount of the annual variation in population density. Thus, the main regulating mechanisms that influence the population dynamics of our studied great snipe population appear to be few and relatively simple.

\section{Acknowledgements}

We are grateful to numerous field assistants and students for help with the data collection and particularly S. L. Svartaas who has joined the field work for the whole study period. We thank O. Enget, Folldal Fjellstyre, who gave us access to the willow grouse data, S. Zwanzig and C. Bandt for comments on the statistical analyses, and M. Dickens, R. Tönjes, P. Rothery and an anonymous reviewer for suggestions that improved this paper. This long-term research project was funded by the Nordic Council, the Research Council of Norway and the Swedish Research Council.

\section{References}

Begon, M., Harper, J.L. \& Townsend, C.R. (1996) EcologyIndividuals, Populations and Communities. Blackwell, Cambridge, MA.

Bergerud, A.T., Peters, S.S. \& McGrath, R. (1963) Determining sex and age of willow ptarmigan in Newfoundland. Journal of Wildlife Management, 27, 700-711.

Berryman, A.A. \& Turchin, P. (2001) Identifying the densitydependent structure underlying ecological time series. Oikos, 92, 265-270.

Bjørnstad, O.N. \& Grenfell, B.T. (2001) Noisy clockwork: time series analysis of population fluctuations in animals. Science, 293, 638-643.

Dennis, B. \& Taper, M.L. (1994) Density dependence in time series observations of natural populations: estimation and testing. Ecological Monographs, 64, 205-224.

Dennis, B., Ponciano, J.M., Lele, S.R., Taper, M.L. \& Staples, D.F. (2006) Estimating density dependence, process noise, and observation error. Ecological Monographs, 76, 323-341.

Einum, S. (2005) Salmonid population dynamics: stability under weak density dependence? Oikos, 110, 630-633.

Fiske, P., Kålås, J.A. \& Sæther, S.A. (1994) Correlates of male mating success in the lekking great snipe (Gallinago media): results from a four-year study. Behavioral Ecology, 5, 210218.

Fox, D.R. \& Ridsdill-Smith, J. (1995) Tests for density dependence revisited. Oecologia, 10, 435-443.

Freckleton, R.P., Watkinson, A.R., Green, R.E. \& Sutherland, W.J. (2006) Census error and the detection of density dependence. Journal of Animal Ecology, 75, 837-851.

Höglund, J., Kålås, J.A. \& Fiske, P. (1992) The cost of secondary sexual characters in the lekking Great snipe (Gallinago media). Behavioral Ecology and Sociobiology, 30, 309-315.

Huffaker, C.B. \& Messenger, P.S. (1964) The concept and significance of natural control. Biological Control of Insect Pests and Weeds (ed. P. DeBach). Chapman \& Hall, London.

Ihaka, R. \& Gentleman, R. (1996) R: a language for data analysis and graphics. Journal of Computational and Graphical Statistics, 5, 299-314.

Jones, P.D., Jonsson, T. \& Wheeler, D. (1997) Extension to the North Atlantic Oscillation using early instrumental pressure observations from Gibraltar and South-West Iceland. International Journal of Climatology, 17, 1433-1450.

Kålås, J.A., Fiske, P. \& Höglund, J. (1997) Food supply and breeding occurrences: the West European population of the lekking Great snipe Gallinago media (Latham, 1787) (Aves). Journal of Biogeography, 24, 213-221.

Lack, D. (1954) The Natural Regulation of Animal Numbers. Clarendon Press, Oxford.

Lande, R., Engen, S., Sæther, B.-E., Filli, F., Matthysen, E. \& Weimerskirch, H. (2002) Estimating density dependence from population time series using demographic theory and life-history data. American Naturalist, 159, 321-337.

Lande, R., Engen, S. \& Sæther, B.-E. (2003) Stochastic Population Dynamics in Ecology and Conservation. Oxford University Press, Oxford.

Leirs, H., Stenseth, N.C., Nichols, J.D., Hines, J.E., Verhagen, R. \& Verheyen, W. (1997) Stochastic seasonality and nonlinear density-dependent factors regulate population size in an African rodent. Nature, 389, 176-180.

Løfaldli, L., Kålås, J.A. \& Fiske, P. (1992) Habitat selection and diet of Great snipe (Gallinago media) during breeding. Ibis, 134, 35-43.

Massoli-Novelli, R. (1988) Status and habitat of the Great snipe in Ethiopia and its movements in Africa. Third Woodcock and Snipe Workshop. 14-16 October 1988. IWRB, Nantes, Paris.

Murdoch, W.W. (1994) Population regulation in theory and practice. Ecology, 75, 271-287.

Newton, I. (1998) Population Limitation in Birds. Academic Press, London.

Pollard, E., Lakhani, K.H. \& Rothery, P. (1987) The detection of density-dependence from a series of annual censuses. Ecology, 68, 2046-2055.

Ricker, W.E. (1954) Stock and recruitment. Journal of the Fisheries Research Board of Canada, 11, 559-623.

Rothery, P., Newton, I., Dale, L. \& Wesolowski, T. (1997) Testing for density dependence allowing for weather effects. Oecologia, 112, 518-523.

Royama, T. (1992) Analytical Population Dynamics. Chapman \& Hall, London.

Rudolf, B., Fuchs, T., Schneider, U. \& Meyer-Christoffer, A. (2003) Introduction of the Global Precipitation Climatology Centre (GPCC), p. 16. Deutscher Wetterdienst, Offenbach am Main.

Sæther, B.-E., Sutherland, W.J. \& Engen, S. (2004) Climate influences on avian population dynamics. Advances in Ecological Research, 35, 185-209.

Shenk, T.M., White, G.C. \& Burnham, K.P. (1998) Samplingvariance effects on detecting density dependence from temporal trends in natural populations. Ecological Monographs, 68, 445-463.

Sugiura, N. (1978) Further analysis of the data by Akaike's information criterion and the finite corrections. Communications in Statistics, Theory and Methods, A7, 13-26.

Turchin, P. (1999) Population regulation: a synthetic view. Oikos, 84, 153-159.

Turchin, P. (2003) Complex Population Dynamics. Princeton University Press, Princeton, NJ.

Wiklund, C.G., Kjellén, N. \& Isakson, E. (1998) Mechanisms determining the spatial distribution of microtine predators on the Arctic tundra. Journal of Animal Ecology, 67, 91-98.

Wolda, H. \& Dennis, B. (1993) Density dependence tests, are they? Oecologia, 95, 581-591. 